\title{
Mortality Following Hip Fracture Surgery During COVID-19 Pandemic Compared to Pre-COVID-19 Period: A Case Matched Cohort Series
}

\author{
De C, MRCS, Harbham PK, MBBS, Postoyalko C, MB ChB, Bhavanasi B, FRCS, Paringe V, FRCS, \\ Theivendran $\mathbf{K}$, FRCS ( $\operatorname{Tr}$ \& Orth) \\ Department of Trauma and Orthopaedics, Sandwell and West Birmingham Hospitals NHS Trust, Birmingham, \\ United Kingdom

This is an open-access article distributed under the terms of the Creative Commons Attribution License, which permits unrestricted use,
distribution, and reproduction in any medium, provided the original work is properly cited
Date of submission: 28th August 2020
Date of acceptance: 13th April 2021

\begin{abstract}
Introduction: This study aims to report on clinical outcomes and 30-day mortality of patients with neck of femur fracture during COVID-19 pandemic and compare the outcomes in a cohort during the same period prior to the pandemic.

Materials and methods: The study included 66 patients with hip fracture over the age of 60 years, presented between 1st March and 15th May 2020 and matched with the patients with hip fractures (75 patients) managed during the corresponding period in 2019 as control. Data was collected on demographics, comorbidities, COVID-19 status, procedures and mortality and complications.

Results: Thirty-day mortality following hip surgery was $13.6 \%$ during COVID-19 pandemic with all the mortalities in patients with ASA Grade 3 and 4. Mortality was considerably high for intracapsular fracture $(20 \%)$ but highest in cemented hemiarthroplasty $(20 \%)$. One third of the hip fractures operated in COVID-19 designated theatre died within 30 days of surgery. Thirty-day mortality rate for COVID-19 positive hip fracture patients were $55.5 \%$. There has been higher 30-day mortality for hip surgeries during COVID-19 pandemic with positive correlation between patient's COVID-19 test status and 30-day mortality following hip surgeries.

Conclusion: There is strong association between 30-day mortality and the designated theatre (Clean/COVID) where the patients were operated on with higher mortality for intracapsular neck of femur fractures with significant mortality associated with cemented hemiarthroplasty particularly among symptomatic or COVID-19 positive patients. Therefore, adoption of a multidisciplinary approach is recommended to optimally balance the risk-benefit ratio for planning of management of hip fractures while considering patient's peri-operative outcomes.
\end{abstract}

Keywords:

30-day mortality, COVID-19, pandemic, hip fracture, preCOVID-19

\section{INTRODUCTION}

Severe acute respiratory syndrome coronavirus-2 (SARSCoV-2) was declared as a pandemic on March 11, 2020 by WHO and by now it has spread to most part of the world'. The pandemic has tested the resilience of health-care systems, including hospitals, which were largely unprepared for the scale of the pandemic ${ }^{2}$. Patients having surgery are a vulnerable group at risk of SARS-CoV-2 (COVID-19) exposure in hospital and might be particularly susceptible to subsequent post-operative complications, due to the proinflammatory cytokine and immunosuppressive responses to surgery ${ }^{3,4}$. Evidence of the safety of performing surgery in COVID-19 exposed hospitals is urgently needed.

Guidelines have been published for the management of surgical patients during the COVID-19 pandemic ${ }^{5,6,7}$ but they are based on limited evidence. Ours is a busy district general hospital which serves more than 500,000 people ${ }^{8}$ in the West Midlands region, delivering its acute trauma and orthopaedic services from two sites. The West Midlands region was considered a hotspot for COVID-19 infection with second highest mortality to London'. Therefore, the impact of COVID-19 on perioperative complications and mortality needs to be established locally in order to enable surgical teams and patients to make evidence-based decisions during this unprecedented pandemic.

The primary aim of this study is to report on clinical outcomes and 30-day mortality rate for neck of femur fracture during COVID-19 pandemic.

Corresponding Author: Chiranjit De, Department of Trauma and Orthopaedics, Sandwell and West Birmingham Hospitals NHS Trust, Sandwell General Hospital, Lyndon, West Bromwich, West Midlands, B71 4HJ, United Kingdom

Email: chiranjit.de@nhs.net 


\section{MATERIALS AND METHODS}

This prospective observational study included all consecutive hip fracture patients over the age of 60 years managed between 1st March and 15th May 2020 (COVID19 cohort). We collected data from the National Hip Fracture Database (NHFD) dataset and from our electronic health records (Cerner). We also collected the demographic data from the same monthly period (1st March to 15th May) in 2019 (pre-COVID-19 cohort) and analysed any demographic differences including age, sex, Nottingham Hip Fracture Score (NHFS), American Society of Anaesthesiologists (ASA) Grade, type of hip fractures and surgical procedures between these two periods. We calculated frailty scores of the patients based on their pre-injury mobility and the level of assistance required. For all the patients, Nottingham Hip Fracture score (NHFS) was calculated and documented. This study was approved and confirmed by the trust's Institutional Review Board that ethical clearance was not required for its conduct. The study was compliant with the STROBE guidelines for reporting observational studies.

Co-morbidities were categorised as numerical values of two, three or more in order to validate NHFS further. We specifically enquired about diabetes, hypertension, respiratory condition, cerebrovascular conditions, renal pathology, cardiological condition, gastrointestinal pathology, neurological conditions and malignancy. Dementia, hormone disorders like hypothyroidism, metabolic disorders, mental health disorders and other conditions which significantly affect the quality of life were considered among the co-morbidities. Operative variables included hip fracture type (Intracapsular or Extracapsular), primary procedure (cemented or uncemented hemiarthroplasty, dynamic hip screw fixation, intramedullary nailing, total hip replacement) and anaesthesia used.

Laboratory testing for COVID-19 infection was based on viral RNA detection by quantitative RT-PCR from swab sampling. Patients were considered to stay in high infection risk zone (Red ward) and planned to be operated in dedicated 'COVID Theatre' using standard personal protective equipment based on either or both clinical and radiological findings. Clinical diagnosis consistent with COVID-19 infection was made by a senior physician and based on clinical presentation indicative of COVID-19 infection, including cough, fever, and myalgia ${ }^{10}$. Radiological diagnosis was based on thorax $\mathrm{CT}$, in keeping with locally implemented protocols. However, all patients suspected with clinical or radiological criteria, subsequently had laboratory testing for COVID-19 infection. Initially due to lack of testing capacity, patient without any suspected clinical or radiological symptoms were not tested. Asymptomatic and COVID-negative patients were managed in 'Blue' wards which were low infection risk zones and were operated in 'Clean' theatre under standard operational protocol as followed during pre-COVID period. However, staffs and surgeons used appropriate tested masks during the procedures. Importantly, all the hip fractures in this situation were discussed in a multidisciplinary meeting involving hip surgeons and orthogeriatric team to formulate an optimal treatment plan with consideration of the available logistics and expertise. Compliance to local guidelines was also ensured and at the same time, patients should get maximal benefit from the surgery by always keeping in mind their risk-benefit ratio.

Continuous variables were expressed as mean \pm standard deviation (SD) and compared using Student's t test. Categorical variables were expressed as percentages and compared using the Chi-squared Test. Independent variables were compared using Unpaired T-Test (Independent T-Test). All statistical tests of significance were two-tailed, and $\mathrm{P}$ values $<0.05$ were considered statistically significant. Statistical analyses were performed using SPSS 16.0 statistics software [SPSS Inc. Chicago, IL, USA]. Kaplan Meier's survival analysis was performed to compare the cohorts with the survival graphs.

\section{RESULTS}

Table I highlights that both groups were similar in terms age, sex, American Society of Anaesthesiology (ASA Grade), Nottingham Hip Fracture Score (NHFS), type of hip fractures and surgical procedures performed between the two cohort as there were no statistically significant differences ( $p$-value $>0.05$ ) and therefore, both the cohorts were closely matched and comparable.

Table II shows higher mortality in the COVID-19 cohort (13.6\%) as compared to the Pre-COVID-19 cohort (5.3\%) and this was statistically significant at p-value $=0.039$ $(<0.05)$. Kaplan Meier Survival analysis depicts statistically significant difference in survival between the Pre-COVID-19 Cohort (red) and COVID-19 Cohort (blue) with log-rank test score $\mathrm{z}=2.07$ and $\mathrm{p}$-value $=0.039(<0.05)($ Fig. 1$)$.

Table II shows distribution of the patients with 30-day mortality with respect to the Nottingham Hip Fracture Score (NHFS), ASA Grading, co-morbidities and pre-injury mobility. It reflects that all the mortalities $(100 \%)$ within 30 days of the surgery were amongst ASA Grade 3 and 4 with statistically significant association between ASA Grade 4 and 30-day mortality with $\mathrm{p}$-value $<0.05$ ( $\mathrm{p}$ value $=0.023$ ). There has been highest mortality $(22.7 \%)$ for those patients having more than three co-morbidities. However, the association of comorbidities was not statistically significant ( $p$ value $=0.277$ ). In our study, we found weak association between pre-injury mobility and 30-day mortality for hip fractures among different categories with p-values $>0.05$ (Table II). 
Table I: Distribution of patients in pre-COVID-19 and COVID-19 cohort with respect to sex, age, ASA grade, NHFS, type of fracture and type of surgery with P-value from corresponding suitable statistical test

\begin{tabular}{|c|c|c|c|}
\hline Variables & Pre-COVID-19 Cohort & COVID-19 Cohort & P-Value \\
\hline Total Patients & 75 & 66 & $0.4907^{\mathrm{a}}$ \\
\hline Male & 20 & 25 & \\
\hline Female & 55 & 41 & \\
\hline Mean Age & 82.7 & 81.3 & $0.275^{\mathrm{b}}$ \\
\hline \multicolumn{4}{|c|}{ Distribution of age (Independent T-Test) } \\
\hline \multicolumn{4}{|c|}{ ASA Grade } \\
\hline ASA-2 & 18 & 14 & $0.7754^{\mathrm{a}}$ \\
\hline ASA-3 & 41 & 33 & \\
\hline ASA-4 & 16 & 19 & \\
\hline \multicolumn{4}{|l|}{ Nottingham Hip Fracture Score (NHFS) } \\
\hline Mean & 5 & 5 & $0.9652^{b}$ \\
\hline \multicolumn{4}{|c|}{ Distribution of NHFS (Independent T-Test) } \\
\hline \multicolumn{4}{|c|}{ Type of hip fractures } \\
\hline Intracapsular & 28 & 30 & $0.6957^{a}$ \\
\hline Extracapsular & 47 & 36 & \\
\hline Total & 75 & 66 & \\
\hline \multicolumn{4}{|l|}{ Type of Hip Surgery } \\
\hline DHS & 21 & 16 & $0.7912^{c}$ \\
\hline IM Nail & 26 & 22 & \\
\hline Cemented Hemiarthroplasty & 14 & 20 & \\
\hline Uncemented Hemiarthroplasty & 4 & 1 & \\
\hline THR & 9 & 3 & \\
\hline Non-operative & 1 & 4 & \\
\hline Total Surgery & 75 & 66 & \\
\hline
\end{tabular}

NB: ASA-American society of anaesthesiology; DHS-dynamic hip screw; IM-Intramedullary; THR-total hip replacement Statistical Tests used: (a) Chi-Square Test; (b) Independent T-Test; (c) ANOVA

Table II: Demographic variable of the study cohort and 30 days mortality

\begin{tabular}{|c|c|c|c|c|}
\hline \multirow[t]{2}{*}{ Variables } & \multicolumn{3}{|c|}{ Distribution of COVID-19 cohort } & \multirow[b]{2}{*}{ P-value } \\
\hline & No of patients & 30 days mortality & $\% 30$ days mortality & \\
\hline \multicolumn{5}{|l|}{ Study Cohort } \\
\hline Pre-COVID-19 & 75 & 4 & 5.3 & 0.039 \\
\hline COVID-19 & 66 & 9 & 13.6 & \\
\hline \multicolumn{5}{|c|}{ Nottingham Hip Fracture Score (NHFS) } \\
\hline NHFS-1 & 1 & 0 & 0 & \\
\hline NHFS-3 & 6 & 0 & 0 & \\
\hline NHFS-4 & 16 & 0 & 0 & \\
\hline NHFS-5 & 18 & 3 & 16.6 & 0.704 \\
\hline NHFS-6 & 18 & 5 & 27.7 & 0.084 \\
\hline NHFS-7 & 5 & 1 & 20 & 0.713 \\
\hline NHFS-8 & 2 & 0 & 0 & \\
\hline \multicolumn{5}{|l|}{ ASA Grade } \\
\hline ASA-2 & 14 & 0 & 0 & \\
\hline ASA-3 & 33 & 3 & 9 & 0.347 \\
\hline ASA-4 & 19 & 6 & 31.5 & 0.023 \\
\hline \multicolumn{5}{|l|}{ No of co-morbidities } \\
\hline 2 & 28 & 2 & 7.1 & 0.277 \\
\hline 3 & 16 & 2 & 12.5 & \\
\hline$>3$ & 22 & 5 & 22.7 & \\
\hline \multicolumn{5}{|l|}{ Pre-injury Mobility } \\
\hline Bed/Wheelchair & 3 & 0 & 0 & \\
\hline Frame & 23 & 4 & 17.4 & 0.573 \\
\hline Two stick & 1 & 0 & 0 & \\
\hline One stick & 17 & 4 & 23.5 & 0.241 \\
\hline Unaided & 22 & 1 & 4.5 & 0.175 \\
\hline
\end{tabular}

NB: NHFS-Nottingham hip fracture score; ASA-American society of anaesthesiology 
Table III: Perioperative variables of the study cohort and 30 days mortality

\begin{tabular}{|c|c|c|c|c|}
\hline \multirow[t]{2}{*}{ Variables } & \multicolumn{3}{|c|}{ Distribution of COVID-19 cohort } & \multirow[b]{2}{*}{ P-value } \\
\hline & No of patients & 30 days mortality & $\% 30$ days mortality & \\
\hline \multicolumn{5}{|l|}{ Type of Fracture } \\
\hline Intracapsular (IC) & 30 & 6 & 20 & 0.169 \\
\hline Extracapsular & 36 & 3 & 8.3 & \\
\hline \multicolumn{5}{|l|}{ Type of surgery } \\
\hline Cemented Hemi & 20 & 4 & 20 & 0.321 \\
\hline Uncemented Hemi & 1 & 0 & 0 & \\
\hline DHS & 16 & 1 & 6.25 & 0.322 \\
\hline IM Nail & 22 & 2 & 9 & 0.446 \\
\hline THR & 3 & 0 & 0 & \\
\hline Non-operated & 4 & 2(both IC) & 50 & 0.028 \\
\hline \multicolumn{5}{|l|}{ Anaesthesia } \\
\hline Regional Anaesthesia & 39 & 3 & 7.7 & 0.496 \\
\hline General Anaesthesia & 18 & 3 & 16.7 & \\
\hline Combined & 5 & 1 & 20 & \\
\hline \multicolumn{5}{|l|}{ Theatre } \\
\hline COVID & 9 & 3 & 33 & 0.023 \\
\hline Clean & 53 & 4 & 7.54 & \\
\hline Not Operated & 4 & 2 & 50 & 0.028 \\
\hline \multicolumn{5}{|l|}{ Ward } \\
\hline Red & 9 & 3 & 33 & 0.043 \\
\hline Blue & 57 & 6 & 10.5 & \\
\hline \multicolumn{5}{|l|}{ COVID Status } \\
\hline Positive & 9 & 5 & 55.5 & 0.008 \\
\hline Negative & 20 & 2 & 10 & \\
\hline Not Tested & 37 & 2 & 5.4 & \\
\hline
\end{tabular}

NB: IC-Intracapsular; Hemi-Hemiarthroplasty; DHS-Dynamic hip screw; IM-Intramedullary; THR-total hip replacement

Table IV: Perioperative variables of the study cohort and 30-day mortality

\begin{tabular}{|lccc|}
\hline Variable & Survivor & COVID-19 Cohort & P-value \\
\hline Mean time to surgery (Hrs) & 43.06 (SD 34.16) & 33.62 (SD 36.72) & 0.218 \\
Anaesthetic induction time (Min) & 15.3 (SD 9.2) & 24.1 (SD 19.5) & 0.083 \\
Procedure duration (Min) & 77.2 (SD 17.3) & 89.3 (SD 23.5) & 0.067 \\
Mean length of stay (Days) & 21.7 (SD 12.75) & 23.44 (SD 13.5) & 0.457 \\
\hline
\end{tabular}

NB: Independent T-Test used to calculate the P-values

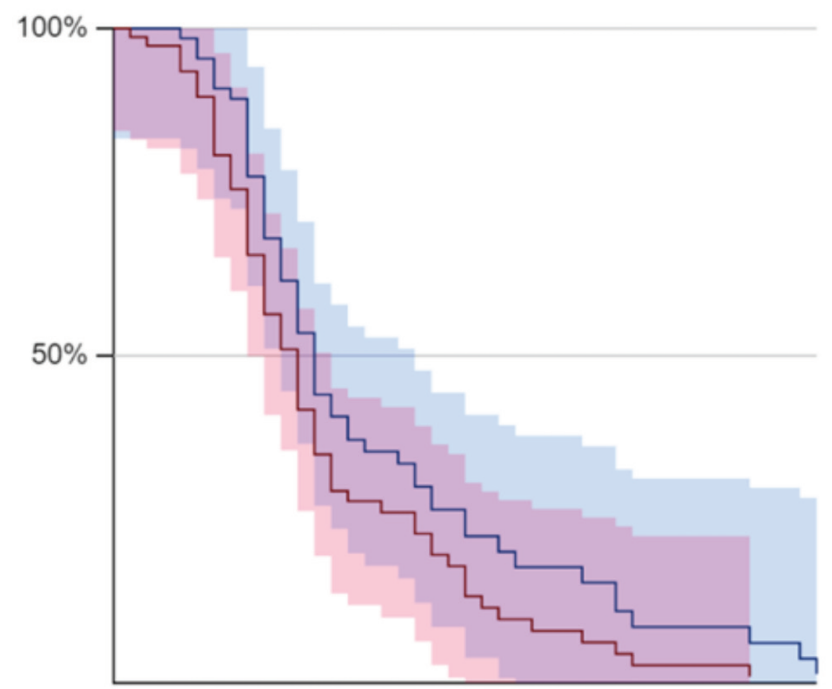

NB: log-rank test score $z=2.07$ and $p$-value $=0.039(<0.05)$. X-axis represents the analysis time and $Y$-axis represents survival probability Fig. 1: Kaplan Meier Survival graph comparing Pre-COVID-19 Cohort (red) and COVID-19 Cohort (blue) 
In our study group (Table III), we had $30(45.5 \%)$ patients with intracapsular neck of femur fracture (IC) compared to $36(54.5 \%)$ patients with extracapsular fracture. Although, mortality was higher for intracapsular fracture, it was not statistically significant ( $\mathrm{p}$ value $=0.169$ ).

Out of these 6 patients with intracapsular fracture, who died within 30 days of surgery, $4(66.6 \%)$ patients were operated with cemented hemiarthroplasty and $2(33.3 \%)$ were not operated being medically unwell for surgery. Out of these 4 patients operated with cemented hemiarthroplasty, $3(75 \%)$ patients were operated in 'COVID theatre' and treated in the 'Red Ward'. However, this higher incidence of death following cemented hemiarthroplasty was not statistically significant $(\mathrm{P}$ value $=0.321)$. Mortalities following extracapsular fracture fixation with dynamic hip screw (P value $=0.322)$ or intramedullary nail $(\mathrm{P}$ value $=0.446)$ were not statistically significant indicating multifactorial causation of post-operative deaths. Although there was higher mortality following use of general anaesthesia, overall mortality was not significantly impacted $(\mathrm{P}$ value $=$ $0.496)$ by the anaesthetic choice. Importantly, there was statistically significant mortality for conservatively managed hip fractures $(\mathrm{P}$ value $=0.028)$.

Table III shows higher mortality for patients operated in 'COVID Theatre' and this association was statistically significant with $\mathrm{P}$ value $=0.023(<0.05)$. Similarly, there was higher mortality for patients who were managed in the 'Red' wards with statistically significant association ( $\mathrm{P}$ value $=$ 0.043).

Table III shows that there was $55.5 \%$ (5 out of 9) mortality for COVID-19 positive patients within 30 days of hip surgery as compared to $10 \%$ (2 out of 10 ) mortality for COVID-19 negative patients. Statistical analysis shows significant association between 30 days mortality and patients' COVID-19 status (positive/negative by COVID-19 $\mathrm{swab})$ with $\mathrm{p}$ value $<0.05$ (P value $=0.008)$.

Table IV shows further analysis of the COVID-19 with respect to peri-operative variables. The patients who died within 30 days were operated sooner (33.62hrs vs $43.06 \mathrm{hrs}$ ) than who survived. However, the anaesthetic induction time, procedure duration and the length of hospital stay were longer for those who died within 30 days of their surgery. These could be an associated finding as these factors were not statistically significant for causation of higher mortality (P-value $>0.05$ ).

When we analyse the cause of death, we found out that 6 $(66.7 \%)$ out of 9 mortalities were due to pulmonary complications. Two patients $(22.2 \%)$ died because of nonpulmonary complications and for one patient $(11.1 \%)$ the cause of death was still under investigation. For all the patients who were COVID-19 positive and died within 30 days of their hip surgery, cause of death was pulmonary complication $(100 \%)$. A total of $50 \%$ of the mortalities within 30 days of hip surgery in COVID-19 negative group was because of pulmonary complications. Considering the number of deaths within 30 days following cemented hemiarthroplasty, pulmonary complication accounted for $75 \%$ (3 out of 4 ) of the mortality.

\section{DISCUSSION}

Since 2007 the NHFD has reported a progressive improvement in mortality in the month after hip fracture. This gives an overall mortality rate of $6.7 \%$ for 2016 . This is better than 7.1\% 30-day mortality in 2015 and continues the steady improvement documented since 2007 when it was $10.9 \%$. Moreover, this trend continues with just 4,007 people (6.1\%) dying in 2018. This figure represents a decrease of one in eight when compared with the mortality figure of $6.9 \%$ the NHFD reported for 2017 and implies that 564 fewer people died within a month of breaking their hip in 2018. Over the seven years (2011-2017) the NHFD recorded that $7.5 \%$ of people died within 30 days. This figure varied significantly across the months of the year, ranging from just $6.7 \%$ in July to a peak of $8.7 \%$ in January ${ }^{11}$. In the study group, $13.6 \%$ patients died within 30 days of their hip surgery which is higher than the national statistics as published in NHFD annual report. However, during COVID19 pandemic there might be additional impact on the overall mortality which might be reflected in the forthcoming NHFD annual report.

In our study group, 30-day mortality according to Nottingham Hip Fracture (NHFS) Score was high compared to predicted 30-day mortality scale according to the NHFS. For NHFS 5,6 and 7, we observed $16.6 \%, 27.7 \%$ and $20 \%$ mortality, respectively within 30 days of hip surgery as compared to $6.9 \%, 11 \%$ and $16 \%$ predicted mortality, respectively ${ }^{12}$.

In the study group, patients who died within 30 days of the surgery, 33\% were ASA Grade- 3 and $66 \%$ were ASA Grade4. Therefore, all the mortalities (100\%) were among ASA Grade 3 and 4 . There has been statistically significant association between patients higher ASA grade and 30-day mortality. However, other studies have found $85 \%$ 30-day mortality for hip fractures for patients with ASA grade 3 and $4^{13}$. Our study population has experienced higher mortality in this group during COVID-19 pandemic compared to the year before the pandemic.

In the COVID-19 cohort, there has been positive correlation between comorbidities and mortality for hip surgeries for neck of femur fracture. We observed higher 30-day mortality for patients with more comorbid conditions and for patients with more than three co-morbidities, the mortality was $22.7 \%$. Although conducted during pre-COVID-19 
pandemic, other clinical studies support that comorbidities were positively associated with surgical procedure and perioperative management of elderly patients aged more than sixty years with hip fracture ${ }^{14}$. However, in our study we found weak association between pre-injury mobility and 30day mortality for hip fractures. This contradicts with studies which shows about $14 \%$ and $18 \%$ mortality within 30 days of hip surgery among patients who were non-ambulatory and frame users, respectively during their pre-injury status and higher correlation with mortality and poor pre-injury mobility $^{15}$. Therefore, it might be other factors during the COVID-19 pandemic which might have impacted the outcome.

According to NHFD-2019 annual report ${ }^{11}$, there was little variation in outcome for intracapsular fracture, but it found significant variation for trochanteric fractures: East of England had the highest percentage of deaths within 30 days (8.5\%) and the North East the lowest (5.9\%). However, in our study we experienced higher mortality for intracapsular neck of femur fractures (20\%) compared to intertrochanteric fractures $(8.12 \%)$. Two third of the patients with intracapsular neck of femur fractures who were operated with cemented hemiarthroplasty died within 30-day of surgery and $75 \%$ of these deaths happened when the surgery was done in dedicated 'COVID-Theatre'. Overall, 20\% of the patients who were operated with cemented hemiarthroplasty died within 30 days of their surgery. Therefore, we experienced the highest mortality for the patients with intracapsular neck of femur fracture operated with cemented hemiarthroplasty in designated 'COVID Theatre'. For management of intertrochanteric fractures, there has been $6.25 \%$ and $9 \%$ mortality within 30 days when they were operated with DHS and intramedullary nailing, respectively. In our study cohort, pulmonary complications have accounted for $75 \%$ of the mortality within 30 days of hip surgery for COVID-19 positive patients who underwent cemented hemiarthroplasty. The exact reason for this remains unknown. We could have hypothesised that the higher mortality following cemented hemiarthroplasty in COVID-19 positive patients may be because of their higher susceptibility to bone cement implantation syndrome (BCIS) due to compromised cardiorespiratory function due to COVID-19 infection. This could act as a 'second hit' to an already compromised physiology. However, multifactorial issue and type of implant or cementation is just one of the multiple factors. Mortality from Bone cement implantation syndrome (BCIS) may vary widely according to the severity. Early mortality in BCIS Grade $1(9.3 \%)$ did not differ significantly from BCIS Grade $0(5.2 \%)$, while early mortality in BCIS Grade $2(35 \%)$ and grade $3(88 \%)$ were significantly higher when compared with Grades 0 and 1. ASA grade III-IV, chronic obstructive pulmonary disease and pulmonary compromise may act as an independent predictor severe BCIS and Severe BCIS was associated with 16 -fold increase in mortality ${ }^{16}$. In our study, management of every hip fracture were discussed in multidisciplinary meeting involving hip surgeons and decisions were taken considering local guidelines, logistics and expertise available. This study was mostly oriented on overall management of hip fractures during the COVID-19 pandemic to figure out any key factors in the practice which could have contributed to the outcome.

There has been statistically significant correlation between 30-day mortality for hip surgery and in which theatre, either clean or COVID which the patients were operated in. As per our local guidelines, we continued to treat patients in 'Red' wards with high infection risk zone who were operated in 'COVID' theatre and therefore the similar mortality pattern followed for their treatment pathway. Hence, we experienced higher mortality for hip fracture surgery in the 'Red ward' (33\%) as compared to the 'Blue ward'(10.5\%). Current evidence from the recent international COVIDSurg Collaborative group ${ }^{17}$ study highlighted overall 30-day mortality in their study was $23.8 \%$, and was high across all patient subgroups with $18.9 \%$ in elective patients, $25.6 \%$ in emergency patients, $16.3 \%$ in patients who had minor surgery, and $26.9 \%$ in patients who had major surgery.

Patients' COVID status (positive/negative by COVID swab) was significantly associated with 30-day mortality following hip surgery with statistically significant positive correlation. Among COVID positive patients with hip fracture, 55.5\% died with 30 days of their hip surgery. The only study so far is a series of cases in Wuhan at an early stage of the pandemic which showed $20.5 \%$ early mortality for across speciality elective surgeries and 35\% mortality for more major surgeries including hip replacement or revision procedures among patient with COVID-19 infection18. However, our study for hip fractures reflects a higher 30-day mortality compared to the current evidence.

In the study we experienced $13.6 \%$ mortality within 30 days of the hip surgery and pulmonary complications have been predominant $(66.7 \%)$ cause for the mortality. Although other studies have indicated 30-day mortality following hip surgery could be $8.7 \%$, the most common causes of death included pulmonary complications, most commonly pneumonia, and concomitant chest infection during admission increases the mortality following the hip surgery ${ }^{19}$. Recently published evidence from multicentre COVIDSurg collaborative study group shows that $38 \%$ mortality within 30 day of surgery and pulmonary complications accounted for $82.6 \%$ of all deaths ${ }^{17}$. However, in the COVIDSurg study there were no comparative (control) group and the number of hip fractures was only 115 over 24 different countries worldwide with various perioperative protocols, testing regimes and health systems. In our study, although with a smaller group, there is a direct comparison with specific perioperative protocols within our hospital avoiding bias and confounding factors. 
In our study, we have noted $50 \%$ mortality for patients who were medically unwell and not fit for surgery. In a study by Moulton et al that mortality at 30 days and one year was $31.3 \%$ and $56.3 \%$, respectively for patients who were managed conservatively for intracapsular neck of femur fracture $^{20}$. The reason for the higher mortality in our series is the morbidity associated with COVID-19 infection.

A recent UK hip fracture study of 18 COVID-19 positive patients over 3 centres showed 30-day mortality rate of $22.2 \%{ }^{21}$. However, in their multi-centres series there was no pre-COVID-19 comparative group. Our series is the only report to date that reports UK hip fractures from a single centre using a control pre-COVID-19 direct comparator group. Therefore, we are confident that the mortality in our series is causally related to COVID-19 mortality rather than other possible confounders of comorbidities, ASA grade and NHFS.

We acknowledge the limitations of a single centre study with relatively small sample size. However, this study reflected early impact of the pandemic on the management of fragility fracture. One has to be cautious about drawing strong conclusions based on this number of patients, but clear trends and observations can be noted, and this may provide helpful guidance when discussing issues of complications including mortality with patients and their family as a part of the consenting process for surgery. The data on delayed complications and revision rates were not available in the short time frame analysed.

\section{CONCLUSION}

We observed a higher 30-day mortality for hip fracture patients during the COVID-19 pandemic. There has been positive association between patient's COVID-19 test results and 30-day mortality following hip fracture surgery. Furthermore, there is strong association with 30-day mortality and in which theatre, Clean or COVID the patients were being operated on. This study also observed higher mortality for intracapsular neck of femur fractures with significant mortality associated with cemented hemiarthroplasty particularly among symptomatic or COVID-19 positive patients. Therefore, adoption of multidisciplinary approach is recommended to optimally balance the risk-benefit ratio for planning of management of hip fractures considering perioperative outcomes.

\section{CONFLICT OF INTEREST}

The authors have no conflict of interest to declare.

\section{ACKNOWLEDGMENT}

We acknowledge contribution of our trauma co-ordinators Michelle Mills, Jennifer Durston and Sushil Satwekar for providing all necessary information from trauma and neck of femur fracture data base. 


\section{REFERENCES}

1. World Health Organization (WHO): WHO announces COVID-19 outbreak a pandemic. 2020. http://www.euro.who.int/en/health-topics/healthemergencies/coronavirus-covid-19/news/news/2020/3/whoannounces-covid-19outbreak-a-pandemic (accessed on 19 April 2020)

2. Horton R. Offline: COVID-19 and the NHS-“a national scandal”. Lancet. 2020; 395(10229): 1022. doi: 10.1016/S01406736(20)30727-3

3. Besnier E, Tuech JJ, Schwarz L. We asked the experts: Covid-19 outbreak: is there still a place for scheduled surgery? "Reflection from pathophysiological data". World J Surg. 2020; 44(6): 1695-8. oi: 10.1007/s00268-020-05501-6

4. Huang C, Wang Y, Li X, Ren L, Zhao J, Hu Y, et al. Clinical features of patients infected with 2019 novel coronavirus in Wuhan, China. Lancet. 2020; 395: 497-506.

5. Coccolini F, Perrone G, Chiarugi M, Marzo FD, Ansaloni L, Scandroglio I, et al. Surgery in COVID-19 patients: operational directives. World J Emerg Surg. 2020; 15: 25. doi: 10.1186/s13017-020-00307-2

6. COVIDSurg Collaborative. Global guidance for surgical care during the COVID-19 pandemic. Br J Surg. 2020; 107(9): 1097103. doi: 10.1002/bjs. 11646

7. Tao KX, Zhang BX, Zhang P, Zhu P, Wang GB, Chen XP, et al. Recommendations for general surgery clinical practice in novel coronavirus pneumonia situation. Zhonghua Wai Ke Za Zhi. 2020; 58(0): E001. doi: 10.3760/cma.j.issn.0529-5815.2020.0001

8. Sandwell and West Birmingham Hospitals. Equality And Inclusion Report. United Kingdom: NHS Trust; 2019. 114 p. https://www.swbh.nhs.uk/wp-content/uploads/2019/03/ED-Annual-Equality-Report-January-2019-FINAL.pdf (accessed on 17 May 2020)

9. Office for National Statistics. Coronavirus (COVID-19). Latest data and analysis on coronavirus (COVID-19) in the UK and its effect on the economy and society. https://www.ons.gov.uk/peoplepopulationandcommunity/healthandsocialcare/ conditionsanddiseases. (accessed on 15 May 2020)

10. Zhou F, Yu T, Du R, Fan G, Liu Y, Liu Z, et al. Clinical course and risk factors for mortality of adult inpatients with COVID-19 in Wuhan, China: a retrospective cohort study. Lancet. 2020; 395(10229): 1054-62. doi: 10.1016/S0140-6736(20)30566-3

11. Royal College of Physicians. National Hip Fracture Database annual report 2019. London: RCP; 2019. https://www.nhfd.co.uk/files/2019ReportFiles/NHFD_2019_Annual_Report.pdf (accessed on 19 April 2020)

12. Moppett IK, Parker M, Griffiths R, Bowers T, White SM, Moran CG. Nottingham Hip Fracture Score: longitudinal and multicentre assessment. Br J Anaesth. 2012; 109(4): 546-50. doi: 10.1093/bja/aes187

13. Yeoh CJC, Fazal MA. ASA Grade and Elderly Patients with Femoral Neck Fracture. Geriatr Orthop Surg Rehabil. 2014; 5(4): 195-9. doi: 10.1177/2151458514560471

14. Wei J, Zeng L, Li S, Luo F, Xiang Z, Ding Q. Relationship between comorbidities and treatment decision-making in elderly hip fracture patients. Aging Clin Exp Res. 2019; 31(12): 1735-41. doi: 10.1007/s40520-019-01134-5

15. Faraj AA, Patil V. Correlation Between Pre-Injury Mobility and ASA Score With the Mortality Following Femoral Neck Fracture in Elderly. Eur J Orthop Surg Traumatol. 2006; 16(2): 130-134. doi: 10.1007/s00590-005-0026-3

16. Olsen F, Kotyra M, Houltz E, Ricksten SE. Bone Cement Implantation Syndrome in Cemented Hemiarthroplasty for Femoral Neck Fracture: Incidence, Risk Factors, and Effect on Outcome. Br J Anaesth. 2014; 113(5): 800-6. doi: 10.1093/bja/aeu226

17. COVIDSurg Collaborative. Mortality and pulmonary complications in patients undergoing surgery with perioperative SARSCoV-2 infection: an international cohort study. Lancet. 2020; 396(10243): 27-38. doi: 10.1016/S0140-6736(20)31182-X

18. Lei S, Jiang F, Su W, Chen C, Chen J, Mei W, et al. Clinical characteristics and outcomes of patients undergoing surgeries during the incubation period of COVID-19 infection. EClinicalMedicine. 2020; 21: 100331. doi: 10.1016/j.eclinm.2020.100331

19. Sheikh HQ, Hossain FS, Aqil A, Akinbamijo B, Mushtaq V, Kapoor H. A Comprehensive Analysis of the Causes and Predictors of 30-Day Mortality Following Hip Fracture Surgery. Clin Orthop Surg. 2017; 9(1): 10-8. doi: 10.4055/cios.2017.9.1.10

20. Moulton LS, Green NL, Sudahar T, Makwana NK, Whittaker JP. Outcome after conservatively managed intracapsular fractures of the femoral neck. Ann R Coll Surg Engl. 2015; 97(4): 279-82. doi: 10.1308/003588415X14181254788809

21. Archer JE, Kapor S, Piper D, Odeh A. The impact of COVID-19 on 30-day mortality in patients with neck of femur fractures. Bone Jt Open. 2020; 1(7): 326-9. doi: 10.1302/2046-3758.17.BJO-2020-0066.R1 